\title{
Correction to: Morphological diversity and connectivity of hippocampal interneurons
}

\author{
Sam A. Booker ${ }^{1,2} \cdot$ Imre Vida $^{3}$ D
}

Published online: 3 April 2019

(C) Springer-Verlag GmbH Germany, part of Springer Nature 2019

\section{Correction to: Cell and Tissue Research https://doi.org/10.1007/s00441-018-2882-2}

The original version of this article inadvertently presented a mistake regarding the termination zones of the entorhinal cotex in the dentate gyrus. The termination zones were erroneously swapped in both Fig. 7 and the associated text.

\section{Text affected:}

\subsection{The Dentate Gyrus (DG)}

The dentate gyrus is the most divergent of the hippocampal subfields with respect to neuronal population, layering and synaptic connectivity. The monopolar dendritic morphology of dentate principal cells, the granule cells (DGC), is found in the molecular layers (ML) and their axons extend from the soma into the hilus. The DG has specific afferents that arise from layer 2 of the lateral and medial EC (outer and middle ML respectively) and the commissural/ associative inputs from hilar mossy cells (inner ML). The output of DGCs is

The online version of the original article can be found at https://doi.org/ 10.1007/s00441-018-2882-2

Sam A. Booker

sbooker@exseed.ed.ac.uk

$\triangle$ Imre Vida

imre.vida@charite.de

1 Centre for Discovery Brain Sciences, University of Edinburgh, Edinburgh EH8 9XD, UK

2 Simons Initiative for the Developing Brain, University of Edinburgh, Edinburgh EH8 9XD, UK

3 Institute for Integrative Neuroanatomy, Charité Universitätmedizin, Berlin, Germany the mossy fiber, which also produces hilar collaterals preferentially targeting INs and the mossy fiber bouton synapse with CA3 PCs (126). The diversity of IN subtypes in the DG is markedly different from that of the CA1-3 regions; albeit with similarities in their principles. Previous morphological studies and reviews have identified at least 4 classes of DG IN, which have distinct morphology $(7,127,128)$. Here we will highlight key differences from CA1-CA3 INs, primarily describing 9 distinct and well established types identified on the basis of anatomical and neurochemical criteria.

\section{Figure affected:}

Revised figure in which "Lateral EC" and "Medial EC" are correctly placed.

Publisher's note Springer Nature remains neutral with regard to jurisdictional claims in published maps and institutional affiliations. 


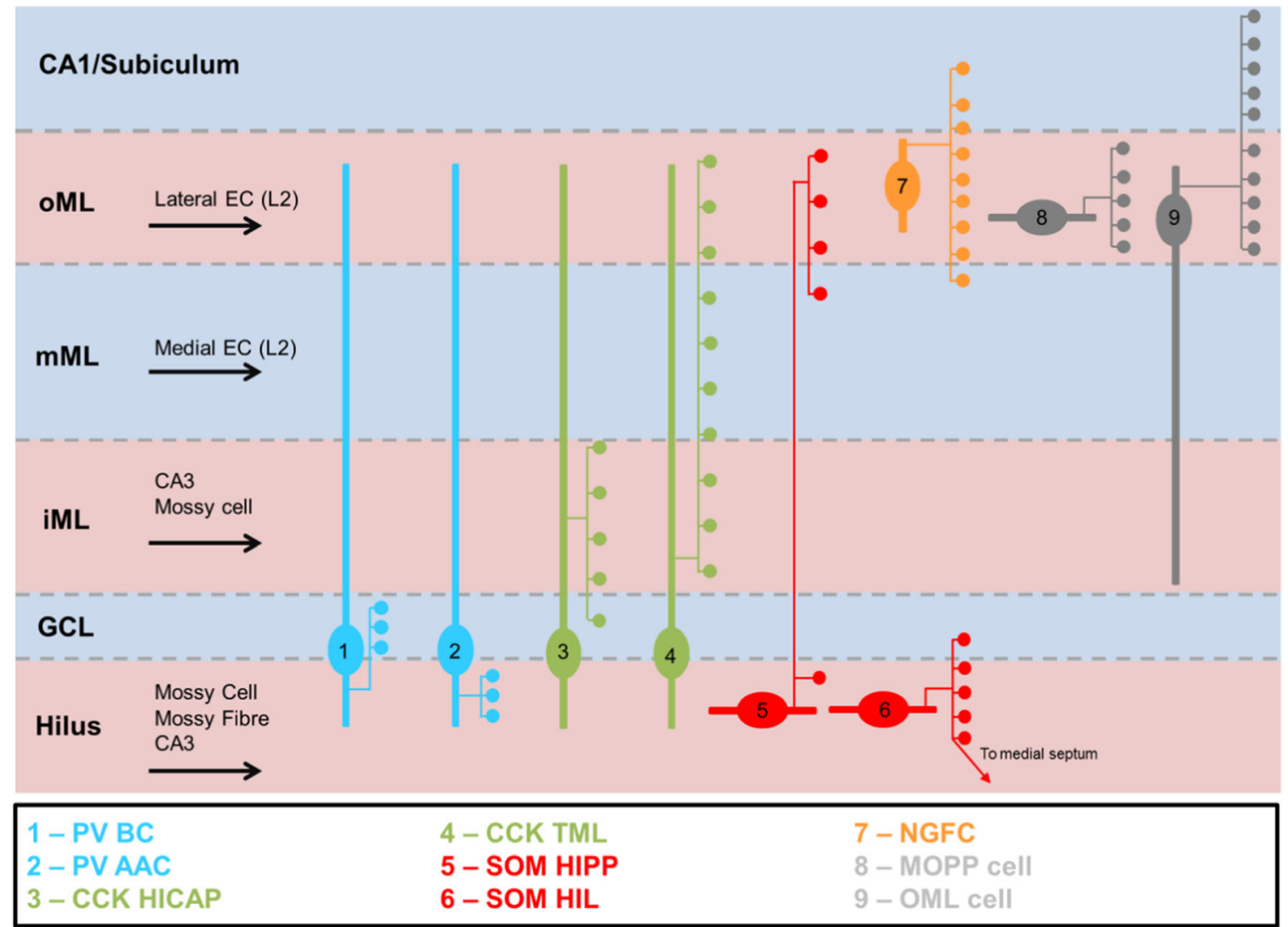

Fig. 7 Summary of identified IN subtypes in the DG. The known morphological and neurochemical subtypes of IN in the DG are depicted, with typical localization of their somata, laminar distribution of their terminal fields (circles) with respect to DG layers (dashed lines): GCL granule cell layer, iML - inner molecular layer, $\mathrm{mML}$ - medial molecular layer, oML - outer molecular layer, hippocampal fissure - thick dashed line 